CLINICAL STUDY

\title{
A randomised controlled clinical trial of antibiotic impregnation of testosterone pellet implants to reduce extrusion rate
}

\author{
S Kelleher, A J Conway and D J Handelsman \\ Department of Andrology, Concord Hospital and ANZAC Research Institute, University of Sydney, Sydney, New South Wales 2139, Australia
}

(Correspondence should be addressed to D J Handelsman; Email: djh@med.usyd.edu.au)

\begin{abstract}
Objective: Testosterone pellet implantation is a safe, effective and convenient form of depot androgen replacement, with extrusion of pellets following about $10 \%$ of procedures the most frequent adverse effect. This study aimed to determine whether extrusion rate could be reduced by antibiotic impregnation of pellets immediately prior to implantation.

Design: Prospective, randomised, parallel-group, open-label study design in a single centre. One hundred and eighty-six androgen-deficient men (400 implantation procedures) were randomised into either a group who had their pellets soaked for $\sim 2$ min in gentamicin solution prior to implantation, or a control group who had the standard implantation procedure.

Methods: Extrusion, infection and/or bruising were evaluated prospectively by self-report from the participants, and retrospectively at subsequent implantation. Other variables (site, shaving, skin preparation, operator, pellet batch, bruising) were collected at implantation time.

Results: The extrusion rate was $20 \%$ lower (odds ratio $=0.80,95 \%$ confidence interval $(\mathrm{CI})$ $0.40-1.62$ ) but not statistically different between the two groups (extrusion rate 23/205 (11.2\%) for the control group vs $18 / 195(9.2 \%)$ for the antibiotic-soak group, $P=0.42)$. One operator experienced more total $(P=0.0002)$ and infection-related $(P=0.0008)$ extrusions and marginally more bruising $(P=0.06)$ than other operators. The operator effect did not appear to be explained by differences in experience or implantation style. There was a 4.6-fold excess (95\% CI 1.6-18.6) of multiple (19 vs 4 expected) over single (22 vs 10 expected) and no (359 vs 386 expected) extrusions. Extrusion was not related to batch number $(P=0.15)$, location $(P=0.15)$, shaving $(P=0.32)$, old or new site $(P=0.59)$, or the presence of suppuration or not $(P=0.42)$; however, povidone-iodine skin disinfectant had statistically fewer extrusions than mixed alcohol solution.

Conclusions: Antibiotic impregnation prior to implantation does not significantly decrease testosterone pellet extrusion rate. An operator effect, not due to experience or procedural style, an excess of multiple extrusions and disinfectant effects were confirmed. Neither location, nor preparation of the site, nor pellet batch, influences extrusion rate.
\end{abstract}

European Journal of Endocrinology 146 513-518

\section{Introduction}

Classical androgen deficiency is characterised by suboptimal physical and mental function, manifest as reduced libido, fatigue, depressed mood and initiative, and loss of bone and muscle mass (1). The goal of testosterone-replacement therapy is to restore the body's androgen exposure to levels comparable with those of eugonadal men by maintaining physiological levels of circulating testosterone (2).

Testosterone-replacement therapy has been available clinically for over five decades. The most widely used formulation over the last three decades has remained testosterone esters in an oil vehicle, despite its pharmacokinetic limitations (3) and painfulness (4). Few new modalities of testosterone delivery have been developed. Transdermal administration of testosterone has gained favour because it is non-invasive, avoids extensive first-pass hepatic metabolism and mimics the diurnal variation in testosterone level observed in eugonadal young men, although it must be applied daily (5) and patches may produce difficulties with poor adhesion and skin irritation $(6,7)$. Other forms of testosterone replacement, such as longer-acting esters (8, 9), microspheres (10), and buccal (11) and sublingual testosterone (12), are still under development.

Among patients who have had experience with other treatment modalities, fused crystalline testosterone pellets are strongly preferred by patients at this centre, according to a previous retrospective review 
(13). The high continuation rates (13) may be attributable to the relative convenience, safety and efficacy of this form of androgen-replacement therapy $(13,14)$. Extrusion of pellets at $\sim 10 \%$ per procedure remains the main adverse event following testosterone pellet implantation $(15,16)$. Extrusion occurs when one or more pellets tunnels back along the s.c. track used for pellet insertion, and exits through the skin, normally through the insertion site. Generally extrusions may be defined as being either early or late in the implantation cycle. Early extrusions are associated with an overt purulent infection at the insertion site that occurs within the first 3 weeks after implantation; late extrusions occur at least 8 weeks after implantation and appear like a small clean blister or a nonpurulent foreign body-like reaction prior to the pellet being extruded. Previous randomised controlled clinical trials have been undertaken to identify contributory factors to the extrusion rate: washing pellets in sterile saline and alcohol solution immediately prior to implantation to remove potentially self-adherent particles (16); and alteration of the site and number of s.c. tracks used for implantation (17). The pelletwashing study evidenced no difference in extrusion rate between the experimental and control groups (16); the site study demonstrated that the number of tracks used for pellet implantation did not affect the likelihood of extrusion but that there was an increased incidence in extrusion in the hip site as opposed to the standard abdominal implantation site (17). The present study was undertaken to assess whether antibiotic impregnation of pellets prior to implantation would decrease the extrusion rate, the premise being that extrusion may be related to an occult infectious process introduced at the time of implantation. To that end, we have undertaken a randomised controlled clinical trial.

\section{Materials and methods}

\section{Design}

The study had a prospective, randomised, parallelgroup unmasked design. Androgen-deficient men with no known allergy to gentamicin and about to undergo routine implantation of testosterone pellets were offered randomisation into groups which differed only in having addition of antibiotic impregnation or not of pellets in gentamicin solution prior to the routine pellet implantation procedure.

The primary outcome variable was extrusion rate per group. The secondary outcome variable was infection and bruising. Procedure-related variables such as site (abdomen vs hip), batch number, operator, subject height and weight, new or old site, type of skin disinfectant used $(10 \% \mathrm{w} / \mathrm{v}$ povidone-iodine $(1 \% \mathrm{w} / \mathrm{v}$ available iodine) (PI) or $95 \%$ ethanol $/ 2 \%$ methanol at $70 \%$ in purified water (EM)), and whether the area was shaved or not prior to implantation were collected.
Outcomes were both evaluated prospectively by subject's self-report (telephone contact was encouraged in the event of an adverse event) and verified retrospectively by systematic questioning of all participants when they returned next for review or treatment. In the event of an infection, participants were advised to return to the department for wound site review and prescription of antibiotics (if needed). In the event of extrusion, participants were asked to record the date and return the extruded remnant.

\section{Experimental intervention}

The use of antibiotics with prostheses to avoid postoperative graft infection is well described (18-20). Gentamicin is an aminoglycoside active against a wide range of pathogenic Gram-negative and Grampositive organisms. If extrusion is caused by a lowgrade infection introduced at the time of implantation, immersion of the pellets in antibiotic could result in local release of high concentration antibiotics in the vicinity of the implants and decrease the infection rate and thus the extrusion rate. Immersion time was determined by two preliminary studies. First, pellets were soaked in a coloured water-based solution over varying lengths of time ( $15 \mathrm{~s}$ to $10 \mathrm{~min})$ and depth of penetration of dyes was evaluated in bisected pellets. Two minutes was considered the optimal duration. Secondly, dry pellets were weighed and then immersed in gentamicin solution for $2 \mathrm{~min}$ to observe the weight gain. Pellets increased in weight $8.8 \pm 0.09 \mathrm{mg}$ (S.E.M.) indicating mean impregnation of $0.32 \pm 0.03 \mathrm{mg}$ gentamicin per pellet.

\section{Subjects}

Subjects were recruited from men routinely attending the department for implantation of testosterone pellets. A detailed explanation of the objective of the study was provided as was an information sheet. If the subject agreed to randomisation they were required to sign an informed consent. The study was approved by the Central Sydney Area Health Service (Royal Prince Albert Hospital) Ethics Review Committee.

\section{Procedures}

Implantations in abdomen or hip site were undertaken as previously described $(13,17)$.

\section{Power and sample size}

The primary end-point was the extrusion rate, for which the standard rate in this centre has been $\sim 10 \%$. Assuming a one-tailed $\alpha$ of 0.05 , a study with 200 per group had a 92, 82 and $53 \%$ power to detect a 75, 66 and 50\% decrease in extrusion rate respectively. 


\section{Data analysis}

Categorical data were analysed by Fisher's exact test using StatXact Version 4 (Cytel Software Corporation, 1998). Continuous data were expressed as means \pm S.E.M. and compared by $t$-tests or ANOVA using SPSS Version 9 (SPSS for Windows, 1997).

\section{Results}

Over a 15 month period, from a possible 411 consecutive procedures, 186 men were randomised in 400 implant procedures with 181 entering the study more than once. Of the 11 procedures in which men were not randomised, three were due to allergy to gentamicin, three were unable to provide informed consent, and five declined randomisation into a study involving prophylactic antibiotic use. The two groups were well matched (Table 1).

Thirty-six men experienced 41 extrusions during the study. There was a non-significant $20 \%$ reduction in the extrusion rate (odds ratio $(\mathrm{OR})=0.80,95 \%$ confidence interval (CI) $0.40-1.62$ ) for the experimental group (18/195 $(9.2 \%))$ as compared with the control group $(23 / 205(11.2 \%))(P=0.31)$ (Table 2). The number of late, non-suppurative extrusions was significantly lower in the experimental group 6/195 (3\%) than the control group $17 / 205(8.2 \%)(P=0.02)$. The number of early, suppurative extrusions was non-significantly higher in the experimental group $(12 / 195(6.1 \%))$ vs the control group (6/205 (2.9\%)) $(P=0.09)$. The rate of infection did not differ between groups $(3 / 195(1.5 \%)$ for the experimental group and
$3 / 205(1.4 \%)$ for the control $(P=0.31))$; the usage of systemic antibiotics did not differ between groups $(6 / 195(3 \%)$ vs $3 / 205(1.4 \%)$ respectively $(P=0.22))$.

There was a significant difference in extrusion rate between two experienced operators and a group of less experienced operators who did fewer than ten procedures each (Table 3). There was no difference between operators in the balance between pre-soak vs control procedures. The most experienced operator had significantly less non-suppurative extrusions and significantly less total extrusions. There were no differences in bruising and infections that did not result in extrusion between the operators (data not shown). Extrusions deviated significantly from a Poisson distribution with a 4.6 -fold excess (95\% CI 1.6-18.6) of multiple (19 vs 4 expected) over single (22 vs 10 expected) and no extrusions (359 vs 386 expected). (Table 3).

Within groups there were statistically less extrusions when PI was used rather than EM for skin disinfection (7/110 PI vs 16/95 EM within the control group $(\mathrm{OR}=0.39,95 \%$ CI $0.13-0.85, P=0.02)$, and $4 / 106$ vs $14 / 89$ for the experimental group $(\mathrm{OR}=0.21$, 95\% CI 0.07-0.66, $P=0.007)$ ). There was no difference between groups in rate of adverse effects whether EM or PI was used as the disinfecting agent to prepare the skin prior to implantation. The hip site implantations had a non-significantly higher rate of extrusion than the abdominal implantations $(21 / 165(12.7 \%)$ vs $20 / 235(8.5 \%), P=0.15)$, and were equivalent in infection rate $(2 / 165$ vs $4 / 235, P=0.63)$; however, preparation of the insertion site (shave/no shave; new/old site) did not influence extrusion, infection or

Table 1 Characteristics of study participants.

\begin{tabular}{lccc}
\hline & $\begin{array}{c}\text { Antibiotic } \\
\text { impregnation }\end{array}$ & $\begin{array}{c}\text { Standard } \\
\text { procedure }\end{array}$ & $\boldsymbol{P}$ \\
\hline$n$ & 195 & 205 & - \\
Age \pm S.E.M. (years) & $45.0 \pm 2.2$ & $46.2 \pm 2.6$ & 0.74 \\
Height \pm S.E.M. $(\mathrm{cm})$ & $176.6 \pm 0.7$ & $177.3 \pm 0.7$ & 0.52 \\
Weight \pm S.E.M. $(\mathrm{kg})$ & $85.2 \pm 1.4$ & $87.7 \pm 1.5$ & 0.22 \\
Body mass index (kg/m $\left.{ }^{2}\right)$ & & & \\
$<20(n)$ & 45 & 51 & 0.18 \\
$20-25(n)$ & 81 & 72 & 0.11 \\
$>25(n)$ & 69 & 82 & 0.19 \\
Hip $(n)$ & 83 & 82 & 0.33 \\
Abdomen $(n)$ & 112 & 123 & 0.61 \\
New scar $(n)$ & 102 & 98 & 0.21 \\
Old scar $(n)$ & 88 & 102 & $0.05^{*}$ \\
Shaved $(n)$ & 98 & 81 & $0.03^{*}$ \\
Unshaven $(n)$ & 97 & 95 & $0.02^{*}$ \\
EM $(n)$ & 89 & 110 & 0.48 \\
PI $(n)$ & 106 & $126(62 \%)$ & 0.48 \\
Hypogonadism & & $79(38 \%)$ & 0.94 \\
$\quad$ Hypergonadotrophic $(\%)$ & $129(66 \%)$ & & 0.94 \\
Hypogonadotrophic $(\%)$ & $66(34 \%)$ & &
\end{tabular}

*Statistically significant. 
Table 2 Rate of adverse events.

\begin{tabular}{lcccc}
\hline & $\begin{array}{c}\text { Antibiotic pre-soak } \\
(n=195)\end{array}$ & $\begin{array}{c}\text { No antibiotic } \\
(n=205)\end{array}$ & $\begin{array}{c}\text { OR } \\
(95 \% \mathrm{Cl})\end{array}$ & $\boldsymbol{P}$ \\
\hline Extrusion & & & & \\
Total & $\mathbf{1 8 ( 9 . 2 \% )}$ & $\mathbf{2 3 ( 1 1 . 2 \% )}$ & $\mathbf{0 . 8 0 ( 0 . 4 0 - 1 . 6 2 )}$ & $\mathbf{0 . 3 1}$ \\
$\quad$ With suppuration & $12(6.1 \%)$ & $6(2.9 \%)$ & $0.46(0.13-1.35)$ & 0.09 \\
$\quad$ Without suppuration & $6(3.1 \%)$ & $17(8.3 \%)$ & $2.80(1.04-9.00)$ & $0.02^{*}$ \\
Infection without extrusion & $3(1.5 \%)$ & $3(1.5 \%)$ & $0.95(0.18-4.76)$ & 0.31 \\
Bruising & $3(1.5 \%)$ & $2(1.0 \%)$ & $0.63(0.10-3.81)$ & 0.47 \\
\hline
\end{tabular}

*Statistically significant.

bruising (data not shown). There was no difference in extrusion rate between 14 different pellet batches used throughout the study $(\mathrm{OR}=0.80,99 \%$ CI $0.42-1.5)$. The rate of extrusion, infection and bruising from both groups remained constant throughout the course of the study (data not shown).

\section{Discussion}

In order to restore androgen exposure, lifelong androgen-replacement therapy needs to be convenient and acceptable whilst maintaining adequate therapeutic levels so as to avoid the deleterious consequences of hypogonadism. Various forms of testosterone replacement have been developed aiming to produce ideal testosterone replacement. Among the alternatives presently available, testosterone pellet implants remain the mainstay of androgen-replacement therapy in this centre due to the long intervals between treatments coupled with few associated adverse effects, the only significant one being pellet extrusion. A previous retrospective survey of nearly 1000 consecutive procedures determined that increased physical activity at work increased the risk of extrusion, and that extrusions deviated from chance in that multiple events were far more likely than by chance, suggesting procedurerelated determinants of extrusion (13). In subsequent controlled randomised clinical trials we identified that a wash-rinse procedure of the pellets to remove surface particles prior to implantation did not decrease extrusion rate (16); nor did changing the site from the traditional abdominal implantation to the hip (17).

The present study demonstrates that the soaking of pellets in antibiotic prior to implantation did not significantly reduce the extrusion rate overall. Furthermore, although the use of the antibiotic resulted in statistically fewer typical extrusions, it was associated with more frequent early infections which resulted in extrusion (Table 2). The reason for this may be that the choice of antibiotic (gentamicin) was effective against Gram-negative (slow-growing) flora, but ineffective against fast-growing Gram-positive flora, which may then have created an environment for a more virulent strain of Gram-positive infection to grow predisposing to early infection and extrusion. The results of this study suggest that the usage of gentamicin may have been appropriate to eliminate slowgrowing Gram-negative bacteria as evidenced by the significantly lower incidence of typical extrusions in the experimental group (Table 2), but less effective against Gram-positive bacteria (predisposing to early extrusion in the experimental group) (Table 2). Additionally it may be that the resultant dosage of gentamicin (mean $1.28 \mathrm{mg}$ per procedure) was too small and the duration of exposure to antibiotics too short to eliminate the Gram-positive infection introduced during pellet insertion.

The increased rate of early infection in the experimental group could theoretically be due to the

Table 3 Extrusion events in relation to operator.

\begin{tabular}{|c|c|c|c|c|c|}
\hline & \multirow[b]{2}{*}{ Total } & \multicolumn{4}{|c|}{ Extrusion $(n)$} \\
\hline & & Total & Multiple & Infected & Non-infective \\
\hline Operator 1 & 169 & $7^{\star}(4.1 \%)$ & 3 & $5^{\star \star}(2.9 \%)$ & $2(1.2 \%)$ \\
\hline Operator 2 & 153 & 25 (16.3\%) & 12 & $11(7.2 \%)$ & $14^{\star \star \star x}(9.1 \%)$ \\
\hline Other operator & 78 & 9 (11.5\%) & 4 & 7 (9.0\%) & $2(2.6 \%)$ \\
\hline Total & 400 & $41(10.2 \%)$ & 19 & $23(5.7 \%)$ & $18(4.5 \%)$ \\
\hline
\end{tabular}


increased risk of contamination brought about by the inclusion of another step in the routine implantation procedure. This is unlikely as one operator experienced significantly more infection-related extrusions than all other operators in both the experimental group and the control group (data not shown), a result not consistent with a general increase in infection due to procedure-related contamination. Therefore, the early infections and extrusions experienced by the experimental group must be due to either failure of the antibiotic to destroy all bacteria, the dose of the antibiotic used being too low to be effective, or non-infective aetiology.

To determine whether the preparation of the implantation site influenced infection or extrusion we collected data such as whether the site was shaved, whether an old scar was re-opened for the implant procedure, and the site of implantation itself. None of these variables affected infection or extrusion. In a previous randomised clinical trial the hip site had a significantly higher rate of extrusion; the reason this has not been found in the present study may be that subjects were able to choose the site of implantation and chose the site that had proved better for them in previous procedures. There was a statistically higher number of extrusions when the disinfectant for the procedure was EM rather than PI; unexpectedly the choice of disinfectant did not influence the implantation site infection rate. This difference in the extrusion rate raises again the possibility of an insidious infectious process predisposing to extrusions.

This study demonstrated that the operator performing the procedure can influence the likelihood of both extrusion and infection. Somewhat unexpectedly, one of the two experienced operators in this study had a lower infection-related extrusion rate and lower total extrusions than other operators (Table 3), and the other more-experienced operator had a higher infectionrelated extrusion rate (in both the control and the experimental groups) than all others (Table 3). Independent observers could not identify systematic procedural differences between operators. The increased likelihood of multiple extrusions, confirming previous findings, and the relationship of operator to extrusion rate suggest a operator/procedure-related cause for extrusion.

The overall extrusion rate for this study $(10.25 \%)$ approximates the extrusion rates of the previous studies conducted in this centre $(13,16,17)$. Whilst gentamicin pre-soak fails to decrease the overall extrusion rate, there is some evidence that it may be effective against slow-growing pathogens which result in 'late' extrusions. A further study trialling either a different antibiotic or a higher dose and/or longer exposure of antibiotic needs to be undertaken and procedurerelated infection-control procedures may warrant further evaluation.

\section{References}

1 Zitzmann M \& Nieschlag E. Hormone substitution in male hypogonadism. Molecular and Cellular Endocrinology 2000161 $73-88$.

2 Handelsman DJ. Androgen action and pharmacologic uses. In Endocrinology, edn 4, vol 3, pp 2232-2242. Eds LJ DeGroot \& LJ Jameson. Philadelphia: WB Saunders Company, 2001.

3 Behre HM \& Nieschlag E. Comparative pharmokinetics of testosterone esters. In Testosterone Action Deficiency Substitution, edn 2, pp 329-349. Eds E Nieschlag \& HM Behre. Berlin: Springer-Verlag, 1998.

4 Mackey MA, Conway AJ \& Handelsman DJ. Tolerability of intramuscular injections of testosterone ester in an oil vehicle. Human Reproduction $1995 \mathbf{1 0} 862-865$.

5 Wang C, Iranmanesh A, Berman N, Steiner MB, Zeil F, Faulkner BM et al. Comparative pharmacokinetics of three doses of percutaneous dihydrotestosterone gel in healthy elderly men - a clinical research center study. Journal of Clinical Endocrinology and Metabolism 199883 2749-2757.

6 Atkinson LE, Chang Y-L \& Snyder PJ. Long-term experience with testosterone replacement through scrotal skin. In Testosterone Action Deficiency Substitution, edn 2, pp 365-388. Eds E Nieschlag \& HM Behre. Berlin: Springer-Verlag, 1998.

7 Meikle WA. A permeation-enhanced non-scrotal testosterone transdermal system for the treatment of male hypogonadism. In Testosterone Action Deficiency Substitution, edn 2, pp 389-422. Eds E Nieschlag \& HM Behre. Berlin: Springer-Verlag, 1998.

8 Behre HN \& Nieschlag E. Testosterone bucuclate (20 Aet-1) in hypogonadal men: pharmacokinetics and pharmacodynamics of the new long-acting ester. Journal of Clinical Endocrinology and Metabolism $1992751204-1210$.

9 Zhang G-Y, Gu Y-Q, Wang X-H, Cui Y-G \& Bremner WJ. A pharmacokinetic study of injectable testosterone undecanoate in hypogonadal men. Journal of Andrology $1998 \quad 19$ $761-767$.

10 Bhasin S, Swerdloff RS, Steiner B, Peterson MA, Meridores T, Galmirini $\mathrm{N}$ et al. A biodegradable testosterone microcapsule formulation provides uniform eugonadal levels of testosterone for 10-11 weeks in hypogonadal men. Journal of Clinical Endocrinology and Metabolism 1992 74 75-83.

11 Dobs AS, Hoover DR, Chen M-C \& Allen R. Pharmacokinetic characteristics, efficacy, and safety of buccal testosterone in hypogonadal males: a pilot study. Journal of Clinical Endocrinology and Metabolism $1996 \mathbf{8 3} 33-39$.

12 Wang C, Ryre DR, Clark R, Kleinberg D, Newman C, Iranmonesh A et al. Sublingual testosterone replacement improves muscle mass and strength, decreases bone resorption, and increases bone formation markers in hypogonadal men - a clinical research center study. Journal of Clinical Endocrinology and Metabolism 199681 3654-3662.

13 Handelsman DJ, Mackey MA, Howe C, Turner L \& Conway AJ. An analysis of testosterone implants for androgen replacement therapy. Clinical Endocrinology $199747311-316$.

14 Zacharin MR \& Warne GL. Treatment of hypogonadal adolescent boys with long acting subcutaneous testosterone pellets. Archives of Disease in Childhood $1997 \mathbf{7 6} 495-499$.

15 Handelsman DJ. Clinical pharmacology of testosterone pellet implants. In Testosterone Action Deficiency Substitution, edn 2, pp 349-364. Eds E Nieschlag \& HM Behre. Berlin: Springer-Verlag, 1998.

16 Kelleher S, Turner L, Howe C, Conway AJ \& Handelsman DJ. Extrusion of testosterone pellets: a randomised controlled clinical study. Clinical Endocrinology 199951 469-471.

17 Kelleher S, Conway AJ \& Handelsman DJ. Influence of implantation site and track geometry on the extrusion rate and pharmacology of testosterone implants. Clinical Endocrinology $20015 \mathbf{5 4}$ $1-7$. 
18 Braithwaite BD, Davies B, Heather BP \& Earnshaw JJ. Early results of a randomized trial of rifampicin-bonded Dacron grafts for extraanatomic vascular reconstruction. Joint Vascular Research Group. British Journal of Surgery $1998 \mathbf{8 5}$ 1378-1381.

19 Hayes PD, Nasim A, London NJ, Sayers RD, Barrie WW, Bell PR et al. In situ replacement of infected aortic grafts with rifampicin-bonded prostheses: the Leicester experience (19921998). Journal of Vascular Surgery 199930 92-98.

20 Huh J, Chen JC, Furman GM, Malki C, King B, Kafie F et al. Local treatment of prosthetic vascular graft infection with multivesicular liposome-encapsulated amikacin. Journal of Surgical Research 1998 74 54-58.

Received 1 August 2001

Accepted 31 October 2001 